\title{
Concentration Polarization, Surface Charge, and Ionic Current Blockade in Nanopores
}

\author{
Dmitriy V. Melnikov, Zachery K. Hulings, and Maria E. Gracheva \\ Department of Physics, Clarkson University, Potsdam, NY 13699
}

(Dated: August 24, 2020)

\section{NEUTRAL PORE AND NEUTRAL PARTICLE}

We first consider tranlocation of the neutral nanoparticle through an uncharged cylindrical nanopore $\left(\sigma_{s}=\right.$ $\left.\sigma_{p}=0\right)$. In this case, $c_{+}=c_{-}=c_{b}$ everywhere, and Eq. (3) of the main text simplifies to

$$
I=-2 c_{b} F D \int_{A_{\perp}} \nabla \phi \cdot \hat{n}_{\perp} d A .
$$

To explicitely isolate contribution to the ionic current due to the particle, we integrate the above equation along the length of the pore and then apply Gauss' theorem to the resulting volume integral on the right:

$$
\begin{gathered}
I L_{p}=-2 c_{b} F D \int_{0}^{L_{p}} d x \int_{A_{\perp}} \nabla \phi \cdot \hat{n}_{\perp} d A_{\perp}= \\
-2 c_{b} F D \oint_{A_{p}} \phi \hat{n}_{\perp} \cdot d \vec{A}-2 c_{b} F D \oint_{a_{p}} \phi \hat{n}_{\perp} \cdot d \vec{a},
\end{gathered}
$$

where the first surface integral is evaluated over the entire surface of the nanopore including caps while the second one is taken over the surface of the nanoparticle. Since the particle is small, it does not perturb the applied electric field near the nanopore surface which remains parallel to the nanopore axis so that the first integral is reduced to $-\Delta V_{p} A_{\perp 0}$ where $A_{\perp 0}$ is the cross-sectional area of the open pore.

Defining $R_{o}=L_{p} /\left(2 c_{b} F D A_{\perp 0}\right)$ as the open pore resistance and relating current $I=\Delta V_{p} / R$ to the blocked pore resistance $R$, we can then write:

$$
\frac{R_{o}-R}{R}=-\frac{\oint_{a_{p}} \phi \hat{n}_{\perp} \cdot d \vec{a}}{\Delta V_{p} A_{\perp 0}} .
$$

Within the same order of accuracy, replacing $R$ and $\Delta V_{p}$ in the denominator of (S3) with their unperturbed, open pore values $R_{o}$ and $\Delta V_{p 0}$ (in which case the electric potential on the surface of the particle $\phi$ is evaluated with respected to the external electric field and is equal to $\phi_{0}$ ) leads to the equation identical to the one derived earlier [1] for a very long pore.

Since the pore's access resistance is unaffected while the particle is confined inside the nanopore and does not stray too close to its ends, we can use for it the open pore value [2] $R_{a}=\left(4 c_{b} F D R_{p}\right)^{-1}$ in order to account for the end effects in the finite length nanopores. To this end, in Eq. (S3), we write $R_{o}$ in terms of the total resistance $R_{\text {tot }, o}=R_{o}+R_{a}$ as $R_{o}=R_{\text {tot }, o} L_{p} /\left(L_{p}+\pi R_{p} / 2\right)$ [3], replace $R_{o}-R$ with $R_{\text {tot }, o}-R_{\text {tot }}$ in the same order of the accuracy, to finally arrive at the equation for the current blockade:

$$
\frac{\Delta I}{I_{o}}=-\eta_{o} \frac{\oint_{a_{p}} \phi_{0} \hat{n}_{\perp} \cdot d \vec{a}}{\Delta V_{p 0} A_{\perp 0}}
$$

where $\eta_{o}=\left[1+\pi R_{p} /\left(2 L_{p}\right)\right]^{-1}$. For small spherical (or ellipsoidal) nanoparticles, the surface integral can be evaluated in the closed form $[1,4]$ leading, for example, for a spherical particle with $\epsilon_{p} \ll \epsilon$ in the limit of a very long nanopore to Eq. (1) of the ain text.

\section{GENERAL CASE OF A CHARGED PARTICLE TRANSLOCATING THROUGH A CHARGED NANOPORE}

In this case $c_{+} \neq c_{-}$, and integration of the current equation (3) of the main text over the pore length gives:

$$
\begin{aligned}
I L_{p} / F D & =-\int_{0}^{L_{p}} d x \int_{A_{\perp}} \nabla \varepsilon \cdot \hat{n}_{\perp} d A_{\perp} \\
& -\int_{0}^{L_{p}} d x \int_{A_{\perp}} c \nabla \phi \cdot \hat{n}_{\perp} d A_{\perp},
\end{aligned}
$$

where $\varepsilon=z_{+} c_{+}+z_{-} c_{-}$and $c=z_{+} c_{-} z_{-} c_{-}$.

We now proceed in the same manner as in SI, Section I. Applying Gauss's theorem to first term on the right of the above equation, we find that for the constant surface charge $\sigma_{p}$ on the particle and assuming the same ionic concentrations at the nanopore ends, this term can be set to zero. In the second term, we replace $c(x)$ with its cross-sectional average value $\bar{c}_{A_{\perp}}(x)$ :

$$
I L_{p} / F D=-\int_{0}^{L_{p}} d x \bar{c}_{A_{\perp}}(x) \int_{A_{\perp}} \nabla \phi \cdot \hat{n}_{\perp} d A_{\perp} .
$$

Keeping in mind that $l_{p} \ll L_{p}$, and averaging $\bar{c}_{A_{\perp}}(x)$ over the particle length, $\left\langle\bar{c}_{A_{\perp}}\right\rangle=l_{p}^{-1} \int_{x_{p}-l_{p} / 2}^{x_{p}+l_{p} / 2} \bar{c}_{A_{\perp}}(x) d x$, we rewrite the above as

$$
\begin{array}{r}
I L_{p} / F D \approx-\int_{0}^{L_{p}} d x \bar{c}_{A_{\perp} 0}(x) \int_{A_{\perp 0}} \nabla \phi \cdot \hat{n}_{\perp} d A_{\perp} \\
-\left\langle\bar{c}_{A_{\perp}}-\bar{c}_{A_{\perp} 0}\right\rangle \int_{x_{p}-l_{p} / 2}^{x_{p}+l_{p} / 2} d x \int_{A_{\perp 0}} \nabla \phi \cdot \hat{n}_{\perp} d A_{\perp} \\
-\left\langle\bar{c}_{A_{\perp} 0}\right\rangle \oint_{a_{p}} \phi \hat{n}_{\perp} \cdot d \vec{a} .
\end{array}
$$


For large nanopores with $R_{p} \gg \lambda_{D}$ at low applied biases, we can use planar approximation for the evaluation of the average net ionic concentration which gives:

$$
\bar{c}_{A_{\perp 0}} \approx 2 c_{b}\left(1+D u_{s}\right),
$$

where the second term corresponds to the enhancement of the ionic concentration due to the nanopore surface change. This contribution is parametrized with help of the nanopore Dukhin number $D u_{s}=\left|\varepsilon_{s}\right| \gamma_{s}, \varepsilon_{s}=$ $\sigma_{s} /\left(c_{b} F R_{p}\right)$, and $\gamma_{s}=-b_{s} / \lambda_{D}+\left[1+\left(b_{s} / \lambda_{D}\right)^{2}\right]^{1 / 2}$ with $b_{s}=2 \epsilon k_{B} T /\left(e\left|\sigma_{s}\right|\right)[5,6]$. Surface charge on the nanoparticle will result in additional increase in the concentration around the nanoparticle which can be parametrized with help of its own Dukhin number $D u_{p}$. However, for large $R_{p}$ and $r_{p}, \bar{c}_{A_{\perp}} \approx \bar{c}_{A_{\perp} 0} \rightarrow 2 c_{b}$, so that Eq. (S7) simplifies to:

$$
\begin{aligned}
I L_{p} / F D \approx-\int_{0}^{L_{p}} d x \bar{c}_{A_{\perp} 0}(x) & \int_{A_{\perp 0}} \nabla \phi \cdot \hat{n}_{\perp} d A_{\perp} \\
& -2 c_{b} \oint_{a_{p}} \phi \hat{n}_{\perp} \cdot d \vec{a} .
\end{aligned}
$$

The last term in this equation is partially responsible for the ionic current change when a particle is in the pore but it produces a current blockade which is independent of the applied volatage (similarly to the neutral particlepore system, see SI, Section I). Note that the surface charge on the particle will actually decrease this contribution by a factor $\sim\left(1-D u_{p}\right)$, owing to the increase in the nanopore conductivity. However, for this effect to be noticeable, the nanoparticle has to be either very small or the surface charge has to be very large which may not always be achievable in practice: For example, for the 5-nm-radius particles with $\left|\sigma_{p}\right|<0.16 \mathrm{e} / \mathrm{nm}^{2}$ discussed in this work, $D u_{p}<0.15$, and as such, it is omitted [7].

To understand the voltage dependence of the current blockade, we consider how the total ionic concentration $\bar{c}_{A_{\perp} 0}(x)$ in the first term of Eq. (S9) changes along the pore axis when a particle moves through it. We start by writing the net ionic flux through the nanopore:

$$
\begin{gathered}
J=F \int_{A_{\perp}}\left(\vec{j}_{+}+\vec{j}_{-}\right) \cdot \hat{n}_{\perp} d A_{\perp}= \\
-F D \int_{A_{\perp}} \nabla c \cdot \hat{n}_{\perp} d A_{\perp}-F D \int_{A_{\perp}} \varepsilon \nabla \phi \cdot \hat{n}_{\perp} d A_{\perp},
\end{gathered}
$$

which after integration along the pore's axis to the left of the nanoparticle position (i. e., for $0<x<x_{p}$ ) can be written as:

$$
\begin{gathered}
J x / F D=-A_{\perp 0}\left[\bar{c}_{A_{\perp 0}}(x)-\bar{c}_{0}\right]- \\
\int_{0}^{x} d x \int_{A_{\perp 0}} \varepsilon \nabla \phi \cdot \hat{n}_{\perp} d A_{\perp},
\end{gathered}
$$

where $\bar{c}_{0}$ is the average net ionic concentration at the nanopore entrance.

Inside the nanopore with the constant charge density $\sigma_{s}$ on its surface, a few Debye lengths away from its ends and the nanoparticle, any inhomogeneities in the electric potential due to the membrane and the nanoparticle charges can be neglected. Then the ionic charge distribution $\varepsilon=z_{+} c_{+}+z_{-} c_{-}$in the direction perpendicular to the nanopore axis is the same, and we can introduce the average ionic concentration induced by the nanopore surface charge in the pore $\bar{\varepsilon}=A_{\perp 0}^{-1} \int_{A_{\perp 0}}\left(z_{+} c_{+}+\right.$ $\left.z_{-} c_{-}\right) d A_{\perp} \sim \varepsilon_{s}$. In this case the second term on the right in Eq. (S11) simplifies, and within the linear approximation in the charge parameter $\varepsilon_{s}$, we can utilize results for the uncharged nanopore [Eq. (S1) and (S4)] to evaluate it. This gives for the average net ionic concentration:

$$
\bar{c}_{A_{\perp 0}}(x)=\bar{c}_{0}-\alpha x, 0<x<x_{p},
$$

and similarly $\left(\bar{c}_{L_{p}}\right.$ is the average net ionic concentration at the nanopore's exit):

$$
\bar{c}_{A_{\perp 0}}(x)=\bar{c}_{L_{p}}-\alpha\left(x-L_{p}\right), x_{p}<x<L_{p},
$$

that is, the average net ionic concentration changes linearly along the nanopore's axis with the constant slope $\alpha$.

By integrating the ionic flux equation (S10) along the whole nanopore length, we can write $\alpha$ with contributons from the nanopore and nanoparticle separated:

$$
\alpha=\frac{\bar{c}_{0}-\bar{c}_{L_{p}}}{L_{p}}-(\langle\bar{\varepsilon}\rangle-\bar{\varepsilon}) \frac{\Delta V_{p} l_{p}}{L_{p}^{2}}+\frac{1}{A_{\perp 0} L_{p}} \oint_{a_{p}} c \hat{n}_{\perp} \cdot d \vec{a},
$$

where $\langle\bar{\varepsilon}\rangle$ is the average ionic charge in the nanopore region between the nanopore's surface and the nanoparticle.

For the charged open nanopore, the last two terms in (S14) vanish, but the concentration gradient is nevertheless non-zero because $\bar{c}_{0} \neq \bar{c}_{L_{p}}$ due to the concentration polarization across the pore (see Fig. S1, the curve for $V_{e}=100 \mathrm{mV}$, for visual representation of this behavior, and SI, Section III for detailed discussion and computational parameters). We can evaluate $\bar{c}_{0}$ and $\bar{c}_{L_{p}}$ just outside the nanopore at a distance of a few $\lambda_{D}$ away from it (where the electrostatic effects from the membrane surface charge become small) by integrating Eq. (S10) with $\varepsilon=0$. Since we need the average concentration value over the cross-sectional area of the pore (shown by the dashed lines in Fig. 1 of the main text), only the part of the net ionic flux will contribute which at a radial distance $r \gtrsim R_{p}$ from the mouth of the pore, is $\approx J\left(R_{p}^{2} / 2 r^{2}\right)$ [8]. As such, the average net ionic conentrations just outside of the nanopore's entrance and exit is related to the net ionic flux $J$ through the nanopore as

$$
\bar{c}_{0}=2 c_{b}-\frac{J \delta}{2 A_{\perp 0} D}, \quad \bar{c}_{L_{p}}=2 c_{b}+\frac{J \delta}{2 A_{\perp 0} D},
$$

where $\delta \sim R_{p} \ll L_{p}$, and these values have to be scaled by $1+D u_{s}$ [as in Eq. (S8)] when entering the nanopore to account for the ionic build up along the charged nanopore surface.

Out of the two terms in Eq. (S14) determining the nanoparticle's effect on the concentration, the first one 
is by far the dominant one. We can see that by estimating the last term as $\sim \Delta c a_{p \perp} /\left(A_{\perp 0} L_{p}\right)$ where $a_{p \perp}$ is the cross-sectional area of the nanoparticle, and the concentration difference between the edges of the particle along the pore's axis is $\Delta c=\bar{c}_{x_{p}+l_{p} / 2}-\bar{c}_{x_{p}-l_{p} / 2} \approx \bar{c}_{L_{p}}-\bar{c}_{0}+$ $\alpha L_{p}$, so that the correction to $\alpha$ is $\sim a_{p \perp} / A_{\perp 0} \ll 1$ and can be neglected. This means that the value of the concentration gradient is smaller than in the open pore case, so that the average ionic concentration at $x<x_{p}$ becomes higher than the open pore value while for $x>x_{p}$, it is lower.

Thus, substituting the average concentrations from Eqs. (S12) and (S13) into the ionic current Eq. (S9), after some calculations, we find that

$$
\begin{gathered}
I L_{p} / F D=\Delta V_{p} /\left(F D R_{o c}\right)-2 c_{b} \oint_{a_{p}} \phi_{0} \hat{n}_{\perp} \cdot d \vec{a}- \\
\frac{1}{2}(\langle\bar{\varepsilon}\rangle-\bar{\varepsilon}) \frac{A_{\perp 0} l_{p} \Delta V_{p}^{2}}{L_{p}^{2}}\left(1-\frac{2 x_{p}}{L_{p}}\right),
\end{gathered}
$$

where $R_{o c}=R_{o} /\left(1+D u_{s}\right)$ is the resistance of the open charged pore [6]. Utilizing further the approach described at the end of SI, Section I, this can be rewritten in terms of the current blockade ratio leading to Eq. (7) of the main text.

\section{NUMERICAL CALCULATIONS}

We validate our results against numerical selfconsistent solution of the Poisson-Nernst-Planck (PNP) equations [Eqs. (4)-(6) of the main text] to find the electric potential and concentration in the system which are then substituted in Eq. (3) of the main text to to evaluate the ionic current. Calculations are performed for a cylindrical nanopore with a cylindrical (or spherical) particle on its central axis. For these calculations, we took $L_{p}=100 \mathrm{~nm}, R_{p}=10 \mathrm{~nm}, c_{b}=100 \mathrm{mM}$ (we use $\mathrm{KCl}$ here, $z_{ \pm}= \pm 1$ ), with applied bias $V$ varying between 100 and $400 \mathrm{mV}$. For these parameters, the EOF contribution to the current is within $5 \%$, and as such, it is omitted. Numerical solution was performed with COMSOL Multiphysics 5.2 using two-dimensional axio-symmetric model. The nanopore was embedded in $\mathrm{SiO}_{2}$ membrane with $100 \times 100 \mathrm{~nm}^{2}$ relaxation reservoirs on both sides. The discretization mesh size varied from $0.2 \mathrm{~nm}$ in the nanopore and its vicinity to $2.3 \mathrm{~nm}$ in the reservoirs away from membrane, while the relative solution tolerance is $10^{-6}$.

Fig. S1 shows the average net ionic concentration $\bar{c}$ in the empty pore. One can see that outside the nanopore, the concentration is above for $x<0$ and below for $x>L_{p}$ the bulk value of $2 c_{b}$, and its value next to the pore ends increases with the applied voltage. It is also seen that the concentration decay to the bulk value is not exponential over a distance $\sim \lambda_{D}(\sim 1 \mathrm{~nm}$ for our parameters $)$, as could be expected from the electrostatic considerations but much slower (in fact, it decreases as $1 /$ distance from
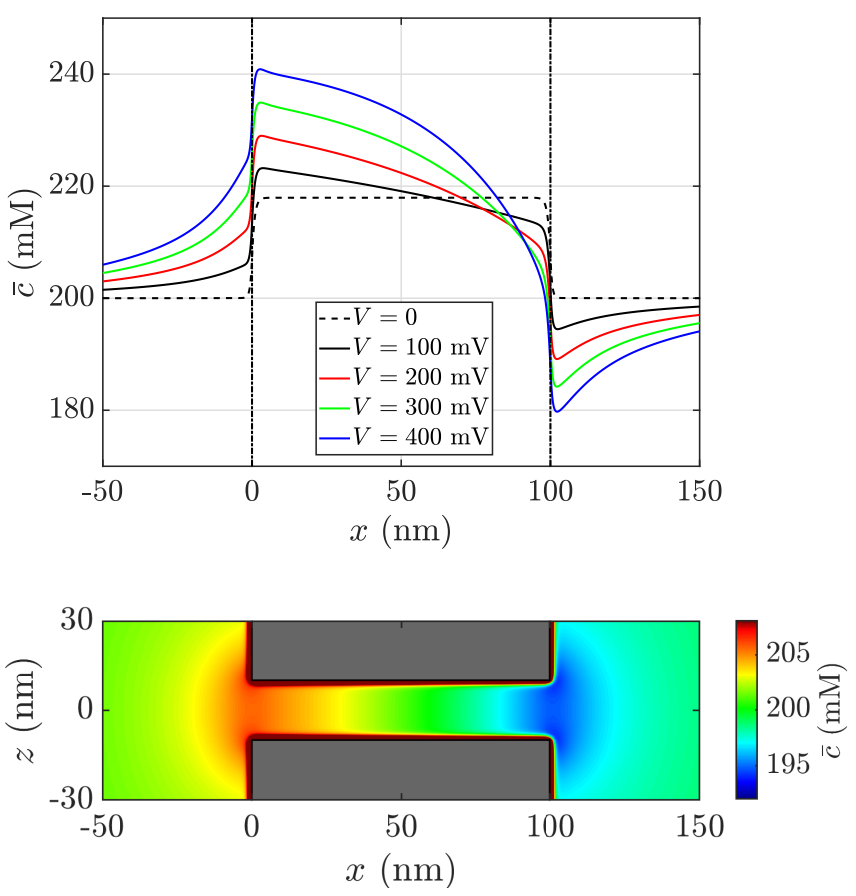

FIG. S1. Top: Average net ionic concentration $\bar{c}$ along the nanopore axis for different applied voltages $V$ in the empty pore. For smaller applied biases, it changes linearly along the nanopore but with increasing $V_{e}$, non-linear deviations appear. Dashed line is $\bar{c}$ at $V=0$ [at equilibrium, see Eq. (S8)]. Bottom: Net ionic concentration distribution at $V=100 \mathrm{mV}$.

the pore to be consistent with the net flux conservation). At $x \sim 0$ and $x \sim L_{p}$, the concentration sharply (over a distance $\sim \lambda_{D}$ ) increases by $\approx 2 c_{b} D u_{s}$ to reach its inpore values [Eq. (S8)]; this change is independent of $V$. While inside the pore, $0<x<L_{p}$, the concentration changes linearly with $x$ for $V=100 \mathrm{mV}$ [in accord with Eqs. (S12)-(S14)], but deviations from the linearity appear when the bias increases.
[1] J. Hurley, Biophys. J 10, 74 (1970).

[2] J. E. Hall, J. Gen. Physiol. 66, 531 (1975).

[3] Z. K. Hulings, D. V. Melnikov, and M. E. Gracheva, Nanotechnology 29, 445204 (2018).

[4] D. C. Golibersuch, Biophys J. 13, 265 (1973).
[5] W. B. Russell, D. A. Saville, and W. R. Schowalter, Colloidal Dispersions (Cambridge University Press, Cambridge, United Kingdom, 1989).

[6] C. Lee, L. Joly, A. Siria, A.-L. Biance, R. Fulcrand, and L. Bocquet, Nano Lett. 12, 4037 (2012). 
[7] For smaller nanoparticles, we can estimate the effect of the enhanced ionic charge near the surface of the particle on the example of a short $\left(l_{p} \ll L_{p}\right)$ cylindrical nanoparticle of radius $r_{p}$ positioned along the central axis of the nanopore (this situation is illustrated in Fig. 1 of the main text). In the planar approximation, with non-overlappind Debye layyers around the nanopore and nanoparticle, we can estimate the average ionic charge density in the region between the particle and nanopore surface as

$$
\left\langle\bar{c}_{A_{\perp}}\right\rangle \approx 2 c_{b}\left(1+\frac{R_{p}^{2} D u_{s}+r_{p}^{2} D u_{p}}{R_{p}^{2}-r_{p}^{2}}\right),
$$

where $D u_{p}=\left|\varepsilon_{p}\right| \gamma_{p}$ is the nanoparticle Dukhin number, $\varepsilon_{p}=\sigma_{p} /\left(c_{b} F r_{p}\right)$ is the nanopore charge parameter, and $\gamma_{p}=-b_{p} / \lambda_{D}+\left[1+\left(b_{p} / \lambda_{D}\right)^{2}\right]^{1 / 2}$ with $b_{p}=2 \epsilon k_{B} T /\left(e\left|\sigma_{p}\right|\right)$. The integrals in the last two terms of Eq. (S7) can be approximated as $\sim \Delta v_{p} R_{p}^{2}$ and $\sim \Delta v_{p} r_{p}^{2}$, respectively, where $\Delta v_{p}$ is the potential drop along the nanoparticle length $\Delta v_{p} \sim \Delta V_{p} l_{p} / L_{p}$ resulting in the overall contribution to the current $\sim 2 c_{b}\left(D u_{p}-1\right) \Delta v_{p} r_{p}^{2}$, that is, the relative current change is smaller than for a neutral particle of the same size by a factor $1-D u_{p}$.

[8] C. T. A. Wong and M. Muthukumar, J. Chem. Phys. 126, 164903 (2007) 